\title{
Identification of the Temporal Components of Seizure Onset in the Scalp EEG
}

\author{
Nora S. O’Neill, Manouchehr Javidan, Zoltan J. Koles
}

\begin{abstract}
Background: The identification of the earliest indication of rhythmical oscillations and paroxysmal events associated with an epileptic seizure is paramount in identifying the location of the seizure onset in the scalp EEG. In this work, data-dependent filters are designed that can help reveal obscure activity at the onset of seizures in problematic EEGs. Methods: Data-dependent filters were designed using temporal patterns common to selected segments from pre-ictal and ictal portions of the scalp EEG. Temporal patterns that accounted for more variance in the ictal segment than in the pre-ictal segment of the scalp EEG were used to form the filters. Results: Application of the filters to the scalp EEG revealed temporal components in the seizure onset in the scalp recording that were not obvious in the unfiltered EEG. Examination of the filtered EEG enabled the onset of the seizure to be recognized earlier in the recording. The utility of the filters was confirmed qualitatively by comparing the scalp recording to the intracranial recording and quantitatively by calculating correlation coefficients between the scalp and intracranial recordings before and after filtering. Conclusion: The data-dependent approach to EEG filter design allows automatic detection of the basic frequencies present in the seizure onset. This approach is more effective than narrow band-pass filtering for eliminating artifactual and other interference that can obscure the onset of a seizure. Therefore, temporal-pattern filtering facilitates the identification of seizure onsets in challenging scalp EEGs.
\end{abstract}

RÉSUMÉ: Identification des composantes temporales du début d'une crise convulsive à l'ÉEG de surface. Introduction: L'identification, à l'ÉEG de surface, des signes les plus précoces d'oscillations rythmiques et d'événements paroxystiques associés à une crise épileptique est très importante pour la localisation du site d'origine de la crise. Dans cette étude, des filtres dépendants des données ont été conçus pour aider à mettre en évidence une activité masquée au début des crises dans les ÉEG problématiques. Méthodes: Des filtres ont été élaborés en utilisant des motifs temporaux communs à des segments sélectionnés de portions pré-ictales et ictales d'ÉEGs de surface. Des motifs temporaux qui expliquaient une plus grande part de la variance dans le segment ictal que dans le segment pré-ictal de l'ÉEG de surface ont été utilisés pour élaborer les filtres. Résultats: L'application des filtres à l'ÉEG de surface a mis en évidence des composantes temporales du début de la crise, qui n'étaient pas évidentes à l'enregistrement ÉEG non filtré. L'examen de l'ÉEG filtré a permis de reconnaître plus tôt le début des crises sur l'enregistrement. L'utilité des filtres a été confirmée qualitativement en comparant l'enregistrement de surface à l'enregistrement intracrânien et quantitativement en calculant les coefficients de corrélation entre les enregistrements de surface et intracrâniens avec et sans filtre. Conclusion: L'approche à l'élaboration de filtres ÉEG selon les données permet la détection automatique des fréquences de base présentes au début des crises. Cette approche est plus efficace que le filtrage passe-bande étroit pour éliminer une interférence due à un artefactuelle ou autre qui peut masquer le début d'une crise. Ce type de filtre facilite l'identification du début des crises dans les enregistrements ÉEGs problématiques.

Can. J. Neurol. Sci. 2001; 28: 245-253

While the clinical symptoms and/or the scalp EEG are useful for the diagnosis of epilepsy, it is the knowledge of the location of the primary seizure focus inside the brain that has the greatest value for the treatment of the disorder. This knowledge is best acquired early in the seizure before the recruitment of susceptible regions. Therefore, the ability to identify the seizure onset at its earliest manifestation in the EEG is desirable. The difficulty, however, is that, in some seizure types, the seizure activity inside the brain begins sometime before it can be discerned in the scalp EEG. It has been suggested that this interval can be of the order of several seconds. ${ }^{1}$ Furthermore, in many cases, the moment of seizure onset in the scalp EEG is obscured by artifacts, swamped by normal background activity or is so subtle that the exact time of seizure onset is difficult to identify. Therefore, the interpretation of the EEG is initially an exercise in seizure onset

From the Department of Biomedical Engineering, University of Alberta, (NSO, ZJK), Department of Neurology, University of Alberta Hospital (MJ), Edmonton, Alberta, Canada.

ReCeived June 9, 2000. ACCEPTED infinAlform May 15, 2001.

Reprint requests to: Z.J. Koles, Department of Biomedical Engineering, 1098 Research Transition Facility, University of Alberta, Edmonton, Alberta, Canada T6G 2V2 
detection, where the objective is to identify the moment when the first sustained temporal pattern associated with seizure activity is recognized amidst the background activity. Identification of the pattern of the temporal activity leading up to this moment would also be informative. There is evidence that the temporal characteristics of the seizure onset, including the frequency and/or the rhythmical components present, also contain information related to the location of the responsible source and the propagation path of the seizure activity. ${ }^{1-11}$ For example, both the hippocampal and neocortical onsets of temporal lobe seizures seem to be characterized by rhythmical waves or patterns with different frequency ranges. These findings suggest that there is value in accurately identifying the onset of ictal activity in the EEG as well as in identifying and observing the detailed time course of the temporal patterns that are present during the seizure onset.

In this paper, a data-dependent temporal filter is described that is very sensitive to temporal changes in the EEG during the seizure onset. Application of the filter usually enables the onset of the seizure to be discerned before it becomes obvious in the raw EEG. In addition, as part of the filter design process, the evolution of distinct temporal components present during the seizure onset can be observed. The design of the filter requires that pre-ictal and ictal segments be selected manually from a single channel in the EEG recording. To do this, the seizure is first visually detected in the EEG and/or from the clinical symptoms of the patient. The ictal segment is then selected early in the seizure period. This is followed by backtracking through the recording until a pre-ictal segment that contains what is judged to be normal background EEG is found. The characteristics of the filter are then derived from temporal patterns present in the two segments. Temporal patterns that account for more variance (signal amplitude) in the ictal segment than in the pre-ictal segment are used to create what is called the temporal pattern (TP) filter. The frequency response of this filter is highly tuned to the seizure and can be considerably more complex than the responses of band-pass filters traditionally used to process the EEG.

The TP-filter is similar but different from other datadependent filters such as the template, matched or inverse filter. Its design is based on such signal processing methods as autoregressive modeling, autocovariance and eigen analyisis. ${ }^{12-21}$ Some very promising seizure detection algorithms using these and related methods have, in fact, been reported in the literature. ${ }^{22,23}$ In contrast to these, the TP-filter is not a seizure detector per se in that the pre-ictal and ictal segments must be manually selected beforehand. The design of the TP-filter does, however, involve the automatic decomposition of the onset into distinct temporal components. The time courses of these components can be associated with either the seizure onset or with background or artifactual activity. Since the TP-filter is specifically tuned to subtle changes in the pre-ictal EEG, it is probably more sensitive to the seizure onset than conventional detectors. Therefore, the TP-filter usually enables the onset to be detected earlier. The spatial patterns in the TP-filtered scalp EEG at the precise moment of seizure onset can serve as input to source localization algorithms such as multiple-signal classification $^{24}$ (MUSIC) and low resolution electromagnetic tomography. ${ }^{25}$ (LORETA).

\section{Methods}

The characteristics of the TP-filter are derived from the temporal patterning of the EEG, at a particular electrode site, in the selected pre-ictal and ictal segments of the recording. If the digital samples from these segments are depicted as $x_{a}(n)$ and $x_{b}(n)$ respectively, where $n=0$ to $T-1$, then the presence of a specific temporal pattern can be detected in these segments by convolving the individual samples with a set of weighting factors that match that pattern. This process is called matched filtering. Mathematically, matched filtering can be represented as:

$$
\begin{aligned}
& y_{a}(n)=\sum_{m=0}^{N-1} p(m) x_{a}(n+m) \\
& y_{b}(n)=\sum_{m=0}^{N-1} p(m) x_{b}(n+m)
\end{aligned}
$$

so that, for example, $y_{a}(0)$ is the weighted sum of $x_{a}(0)$ to $x_{a}(N-1), y_{a}(1)$ is the weighted sum of $x_{a}(1)$ to $x_{a}(n)$ and so on. Therefore, from $T$ samples, $T-N+1$ values of $y_{a}(n)$ and $y_{b}(n)$ can be computed using the $N$ factors in the temporal pattern $p(m)$. Strictly, $y_{a}(n)$ and $y_{b}(n)$ are match-filtered versions of $x_{a}(n)$ and $x_{b}(n)$ but they can also be thought of as temporal components of $x_{a}(n)$ and $x_{b}(n)$.

For the TP-filter, the temporal patterns are chosen to emphasize the seizure onset activity and de-emphasize the background or artifactual activity. This is accomplished by selecting these patterns so that the variance of $y_{b}(n)$ (derived from the ictal segment) is large compared to variance of $y_{a}(n)$ (derived from the pre-ictal segment). Here, the term variance is used in the purely statistical sense to mean a measure of the magnitude variations of the numbers in each of these components. It can then be said, for example, that the pattern $p(m)$ accounts for more variance in the ictal segment than in the pre-ictal segment. There are an infinite number of temporal patterns that will result in differences of this kind as some of the patterns will only be marginally different from one another. Therefore, the temporal patterns are chosen so that they account for components that are independent.

Two temporal components $y_{1}(n)$ and $y_{2}(n)$ are said to be independent if

$$
\sum_{n=0}^{T-N+1} y_{1}(n) y_{2}(n)=0
$$

so that independent components have a covariance of 0 . If $y_{1}(n)$ and $y_{2}(n)$ are derived from $x_{a}(n)$, they are called independent temporal components of $x_{a}(n)$. It can be shown that both $x_{a}(n)$ and $x_{b}(n)$ can have up to $N$ mutually independent components. For the TP-filter, the temporal patterns are chosen so that they simultaneously account for independent components in both the selected pre-ictal and ictal segments. They are therefore called common temporal patterns. Those common temporal patterns that, in terms of their corresponding independent temporal components, account for more variance in the ictal segment than in the pre-ictal segment are used to form the TP-filter. The impulse response, $h(m)$ of this filter is a weighted sum of these patterns. That is:

$$
h(m)=\sum_{i=1}^{q} c_{i} p_{i}(m)
$$


where $p_{i}(m), i=1$ to $q$, are the $q$ common temporal patterns that account for more variance in the ictal segment than in the pre-ictal segment and where the $c_{i}$ are the associated weighting factors. These weighting factors are determined by finding the best fit, in a least squares sense, of the $q$ independent temporal components to the ictal segment. That is:

$$
x_{b f}(n)=\sum_{i=1}^{q} c_{i} y_{b i}(n)
$$

This fitting procedure leads to $x_{b f}(n)$, the TP-filtered version of $x_{b}(n)$. In equation (4), $y_{b i}(n)$ are the $q$ independent components of $x_{b}(n)$. Using equation (1b), it can be shown that:

$$
x_{b f}(n)=\sum_{m=0}^{N-1} h(m) x_{b}(n+m)
$$

where the impulse response of the filter, $h(m)$, is given by equation (3).

Once derived from the selected pre-ictal and ictal segments, the TP-filter is applied to all channels in the EEG recording. The details of the method used to obtain the patterns, $p_{i}(m)$, and the weightings on these patterns, $c_{i}$, are given in the appendix.

\section{RESULTS}

The tracings in Figure 1, all of which contain a seizure, demonstrate the performance of the TP-filter. Tracings 1 to 3 are the raw EEGs while tracings 4 to 6 are their TP-filtered counterparts. Tracing 1 is a simulated EEG while tracings 2 and 3 are single-channel excerpts from actual clinical EEGs.

The simulated EEG of tracing 1 was constructed from several distinct sinusoidal components. As background, 0.30, 0.35, 1.0, and $7 \mathrm{~Hz}$ components were superposed and this composite signal is present throughout the tracing. Frequencies of $4 \mathrm{~Hz}$ and $2.5 \mathrm{~Hz}$ were used to simulate the seizure with the former component starting at about $5.5 \mathrm{~s}$ and the latter at about $7.5 \mathrm{~s}$. The TP-filtered version of this tracing shows that the background activity has been eliminated, the moment of seizure onset has been sharpened and that the two frequencies in the seizure are more obvious.

With regard to the clinical EEGs in Figure 1, we note that the onset of the seizure in tracing 2 occurs at about $5 \mathrm{~s}$ while, in tracing 3, the moment of onset is more obscure. The TP-filtered versions of these tracings contain far less muscle artifact than the raw versions. After filtering, it is clear that in the pre-ictal interval, there are frequency components present that are also present in the ictal interval. This is particularly obvious in the filtered version of tracing 3 . In this tracing, the seizure activity appears to grow out of the pre-ictal segment. Using the criterion that the seizure onset occurs at the moment when persistent ictal activity begins, the onset, in this filtered EEG, occurs at about $3.5 \mathrm{~s}$. This is a much earlier point in time than that suggested by the raw tracing.

Figures 2 to 9 illustrate, in some detail, the design and the performance of the TP-filter. The results were obtained from a 64 channel EEG consisting of simultaneous scalp, depth and subdural recordings from a patient with refractory complex partial seizures. This EEG was selected because the seizure onset was not obvious in the scalp tracings until some time after it was clear from the intracranial tracings that the onset had occurred. The locations of the depth and subdural electrodes are shown in
Figure 2 and the raw EEG containing the seizure is shown in Figure 3. The recording is referential with respect to the left ear electrode A1. Figure 3 suggests that the seizure onset occurs at about 5 or $6 \mathrm{~s}$ into the recording and that it originates near depth electrode a 2 and subdural electrode $\mathrm{c} 4$. At 7 or $8 \mathrm{~s}$ into the recording, there is evidence of the seizure at the scalp electrodes. However, this would probably not have been recognized as such in the absence of the intracranial recordings. To demonstrate the performance of the TP-filter, four depth electrodes (a2 - a5) in the left-temporal lobe, two subdural strip electrodes (c4 - c5) located under the inferior side of the left anterior temporal lobe, and six scalp electrodes, located at standard 10 - 20 sites over the left hemisphere, were used.

The segment of the EEG selected from Figure 3 for analysis is shown in Figure 4. In addition, the tracings in Figure 4 have been band-pass filtered into the range 1-55 Hz. This figure confirms that the seizure has a left hippocampal onset localized to the inferomedial depth (a2) and subdural (c4) electrode sites and suggests that the onset of the seizure actually occurs at about 5.2s.

To design the TP-filter, the pre-ictal and ictal segments were selected as shown at the top of Figure 4. Four hundred digital samples ( 2 s) from each segment at F9 were used to calculate the temporal patterns common to the two segments. The independent components of the tracing from F9 corresponding to the 15 common temporal patterns that account for more variance in the ictal segment than in the pre-ictal segment are shown in Figure 5. The numbers along the right-hand side of the tracings in Figure 5 indicate how the total variance in the pre-ictal and ictal segments is accounted for by each of these patterns. These numbers are the eigenvalues defined by equation (A5) in the appendix. For example, Figure 5 shows that, of the total variance accounted for by the common temporal pattern underlying component 1, 97\% comes from the ictal segment. Therefore, only $3 \%$ comes from the pre-ictal segment. This division of variance can be seen by comparing the amplitudes of the preictal $(3-5 s)$ and ictal segments $(7-9 s)$ in component 1 . As the eigenvalues decrease from $97 \%$ to $50 \%$, relatively less and less of the total variance accounted for by the underlying temporal pattern comes from the ictal segment.

An examination of the components in Figure 5 also indicates that, in terms of their waveforms, several pairs are very similar. Each of the pairs contains distinct frequencies with distinctly modulated amplitudes. For example, components 1 and 2 are both very similar in terms of their frequencies $(14-15 \mathrm{~Hz})$ and amplitude modulations. A closer examination, however, reveals that the two components are in fact shifted in phase from one another by $90^{\circ}$. This is true for components 3 and 4 where the frequency is also about $14-15 \mathrm{~Hz}$, for components 5 and 6 where the frequency is about $7-8 \mathrm{~Hz}$, for components 7 and 8 where the frequency is about $18 \mathrm{~Hz}$ and so on. Of all the components in Figure 4, only 9, 10 and 13 appear to stand alone.

Given the distinct frequencies evident in the independent components of Figure 5, the selected pre-ictal and ictal segments from F9 and from $\mathrm{c} 4$ and a2 were subjected to Fourier analysis. The results of this analysis are shown in Figure 6. The frequency spectra show that, in the ictal segment, both intracranial tracings contain strong components at $7.2 \mathrm{~Hz}, 14.5 \mathrm{~Hz}$, and $21.5 \mathrm{~Hz}$. Because the second and third of these frequencies are multiples 


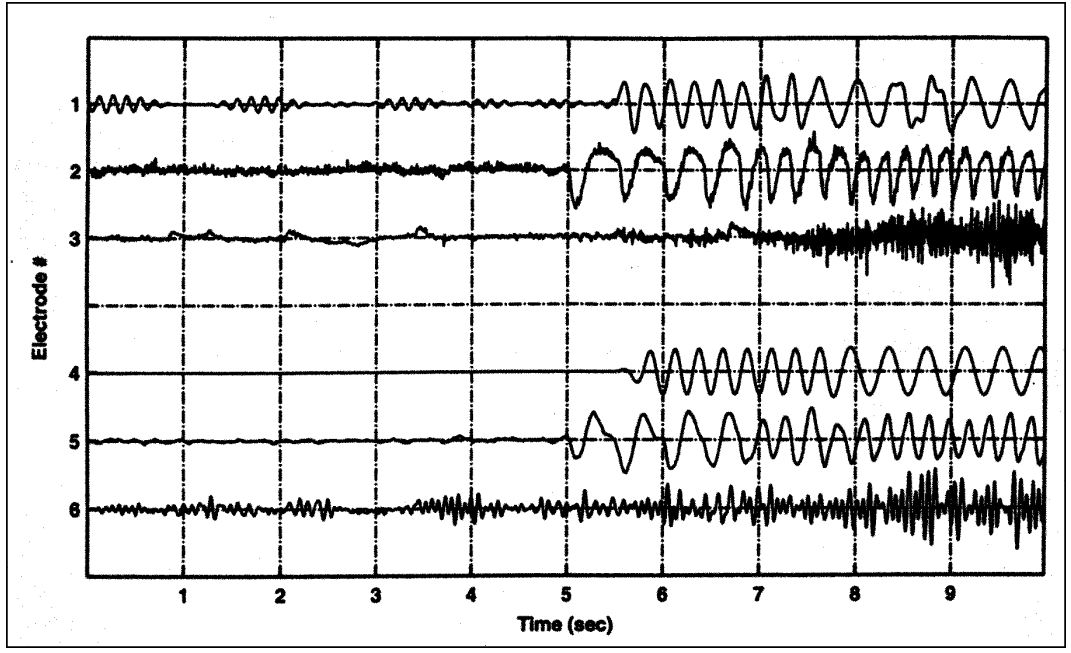

Figure 1: Tracings 1 to 3 are the raw EEGs while tracings 4 to 6 are their TP-filtered counterparts. Tracing 1 is a simulated EEG while tracings 2 and 3 are single-channel excerpts from actual clinical EEGs. All three tracings contain a seizure.

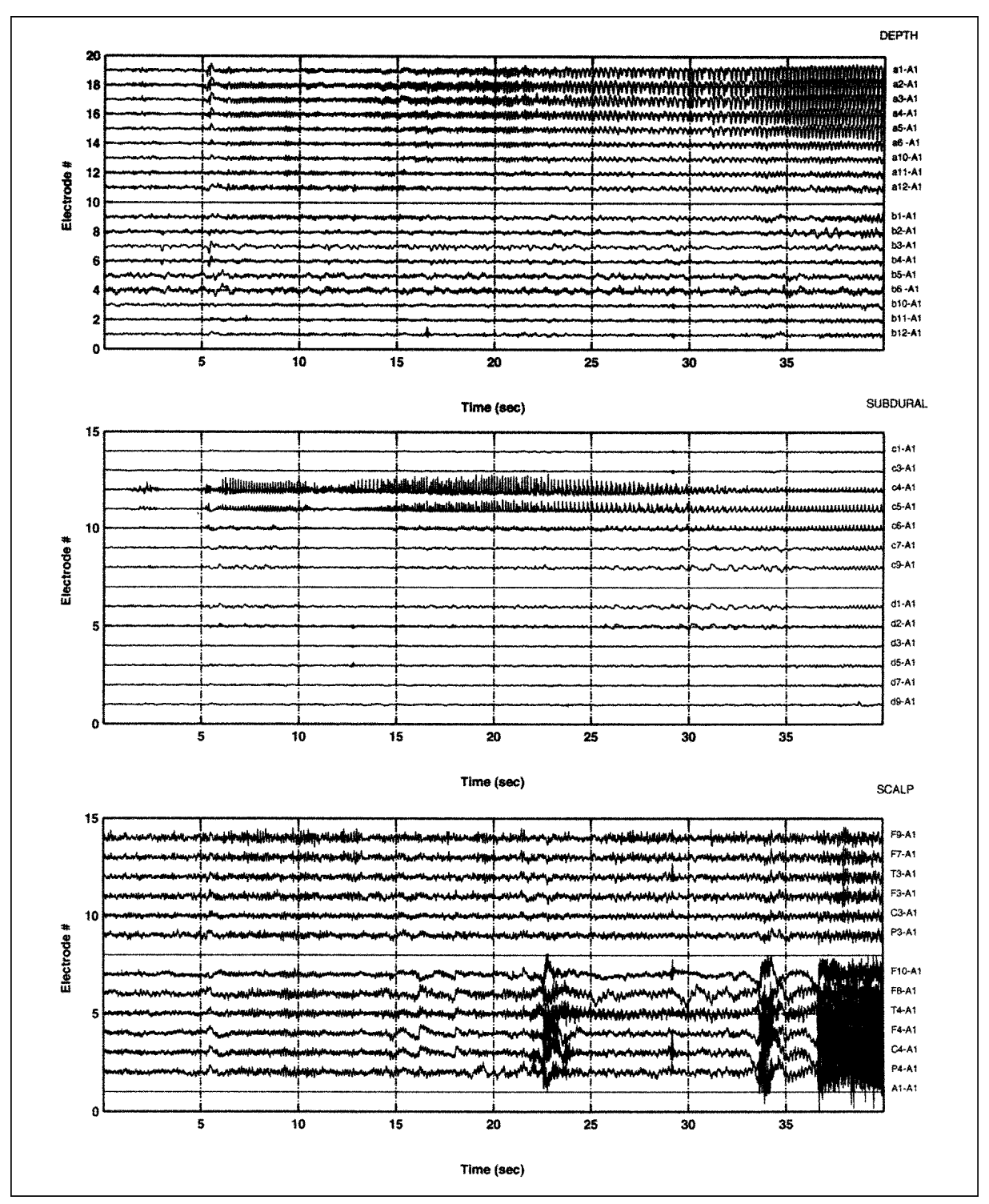

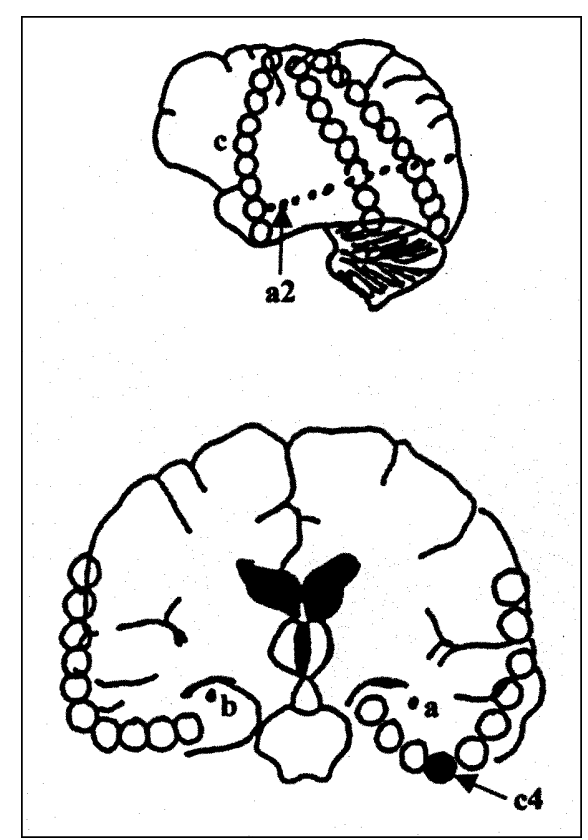

Figure 2: The depth and subdural electrode locations with depth-electrode a2 and subdural-electrode c4 shown. Electrode locations were obtained from MR images of the patient.
Figure 3: The raw 64 channel EEG containing a seizure. All tracings were obtained with respect to a common electrode at A1. The top panel shows the tracings from the depth electrodes, the middle panel from the subdural strip electrodes and the bottom panel from the scalp electrodes. 


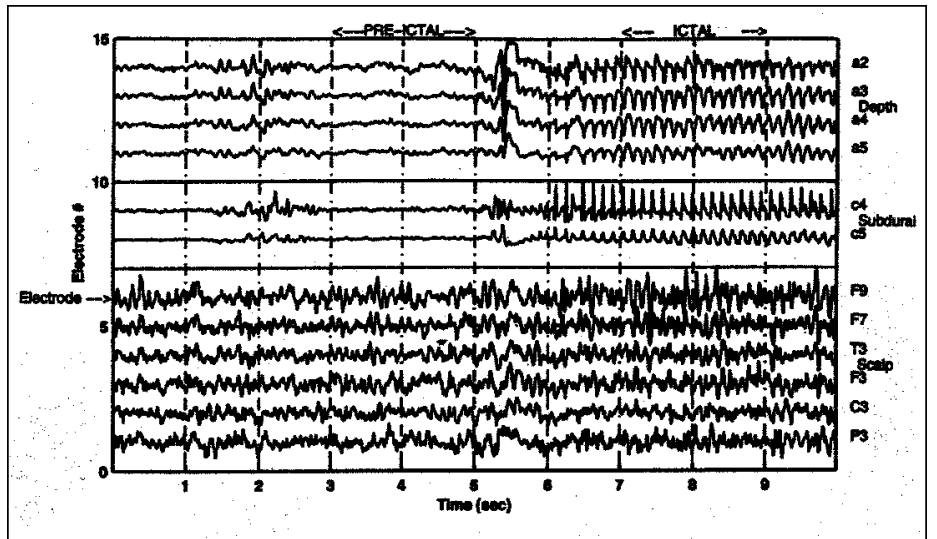

Figure 4: The EEG segment used to demonstrate the temporal-pattern filters. Tracings labeled a 2 to a5 are from the depth electrodes, $c 4$ and c5 are from the subdural strip electrodes and F9, F7, F3, T3, C3 and P3 are the scalp electrodes. Electrode F9 is used to design the TP-filters. The pre-ictal and ictal segments used for the design of the filters are shown along the top of the graph.

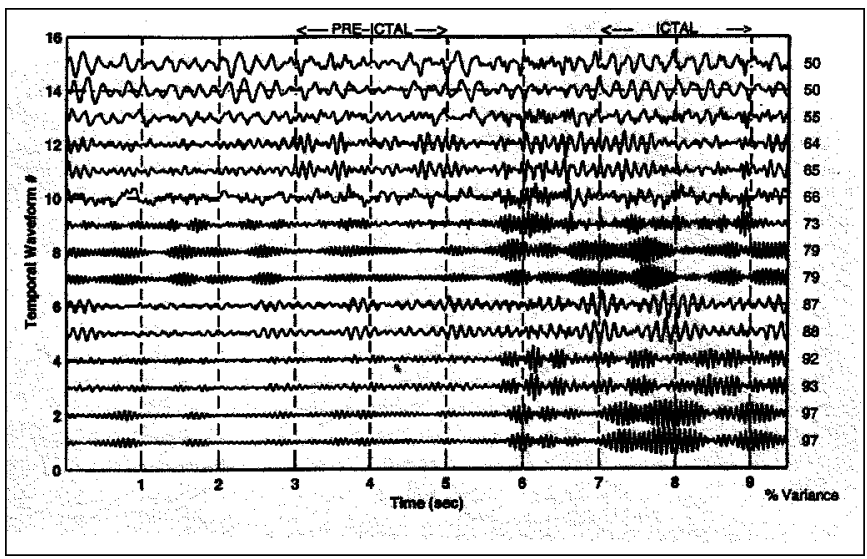

Figure 5: The temporal waveforms resulting from the decomposition of the recording from electrode F9 in Figure 4 using the temporal patterns common to the pre-ictal and ictal segments. Each number along the right-hand vertical axis of the graph is the percentage of the total variance in the pre-ictal and ictal segments, accounted for by one of the common temporal patterns, that comes from the ictal segment.

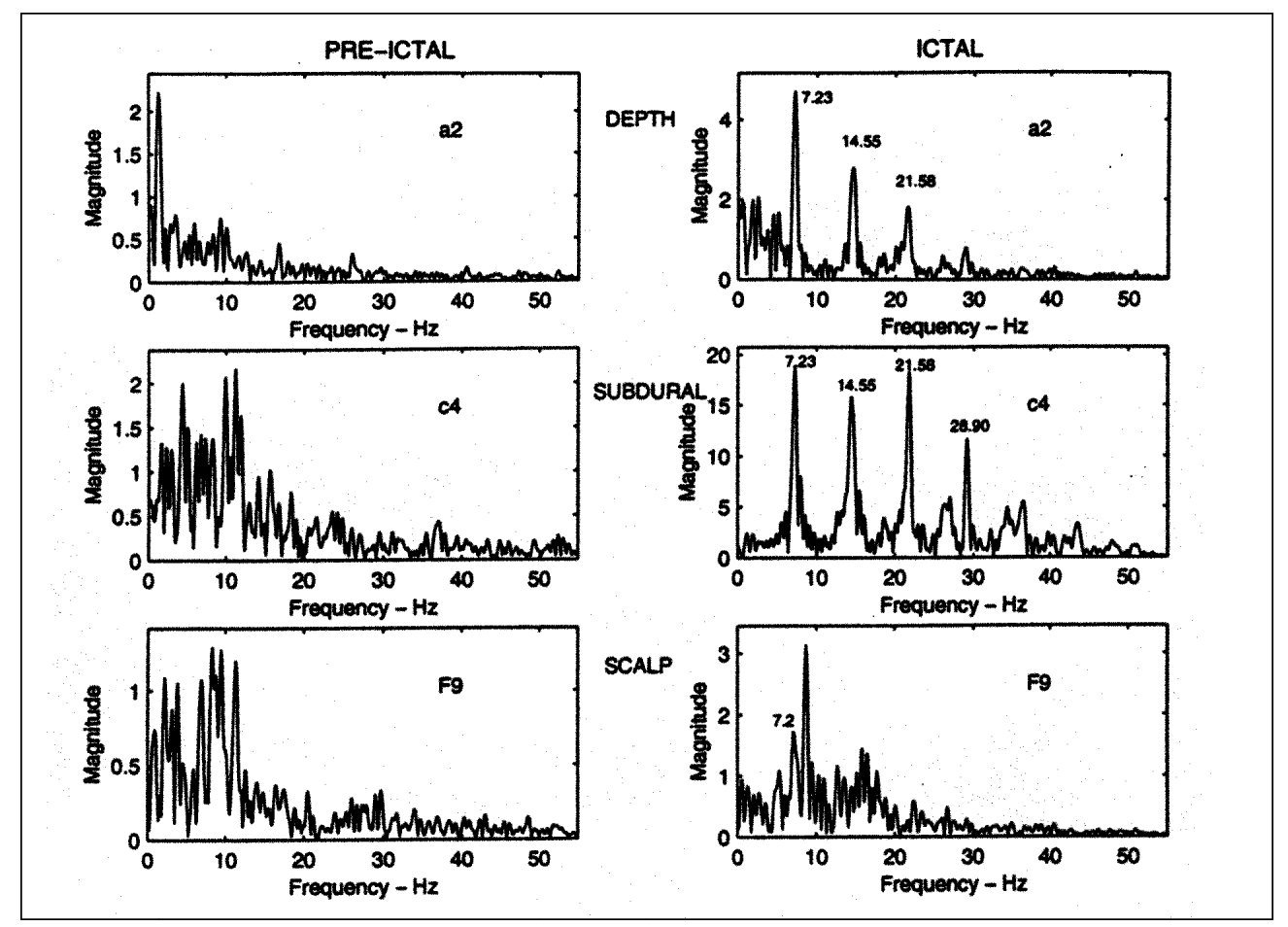

Figure 6: The frequency spectra of the pre-ictal and ictal segments of the depth (a2), subdural (c4) and scalp recordings $(F 9)$ respectively.

of the first, these components could be harmonically related. Peaks at these frequencies are not obvious in the spectrum at F9 during the ictal segment nor are they obvious in any of the spectra in the pre-ictal segment. However, a closer examination of the spectrum from F9 in the ictal segment shows that there is in fact a peak at $7.2 \mathrm{~Hz}$ and that this peak is actually the second highest in the spectrum. It would appear, therefore, that the seizure onset at F9 is swamped by background activity. The independent components in Figure 5 clearly indicate that frequency components at $7.2 \mathrm{~Hz}$ and $14.5 \mathrm{~Hz}$ are in fact present at F9 and that changes in the amplitudes of these components are related to the onset of the seizure.

Figure 7 shows the EEG of Figure 4 after the application of the TP-filter designed from the common temporal patterns underlying independent components 1 to 4 in Figure 5. As expected, all of the tracings in the filtered EEG contain a strong 14-15 Hz frequency component. Figure 8 shows the EEG of Figure 4 after the application of the TP-filter designed from the 
common temporal patterns underlying independent components 5 and 6 in Figure 5. All of the tracings now contain a strong 7-8 $\mathrm{Hz}$ frequency component. We note that the seizure onset is clearer in the scalp tracings of Figure 7 than it is in the scalp tracings of Figure 8 and that it is most evident at electrode F9, the scalp electrode site closest to the depth and subdural electrode sites.

The frequency responses of the TP-filters used to obtain Figures 7 and 8 from Figure 4 are shown in Figures 9A and B respectively. These were obtained by Fourier transformation of the respective impulse responses of the filters. In Figure 9A, the major peaks in the frequency response are at 12.9 and $15.9 \mathrm{~Hz}$. Given these frequencies, a more careful examination of the tracings in Figure 7 indicates that, in fact, frequencies between about 13 and $16 \mathrm{~Hz}$ are indeed present at various times. This filter is therefore labeled the TP-14 filter. In Figure 9B, the three largest peaks in the frequency response are at about 7.7, 15.8 and 19.4 Hz. The $7.7 \mathrm{~Hz}$ peak is, however, more than twice as high as the $15.8 \mathrm{~Hz}$ peak. This is probably why all of the tracings in Figure 8 appear to have only a 7-8 Hz component. This filter is therefore labeled the TP- 8 filter. The composite frequency response of the TP- 8 and TP-14 filters, obtained from the temporal patterns underlying independent components 1 to 6 in Figure 5, is shown in Figure 9C and is labeled as the TP-8-14 filter.

To determine whether filters with frequency responses as elaborate as those shown in Figures $9 \mathrm{~A}$ and $\mathrm{B}$ are required to detect the onset of the seizure in the scalp EEG, the tracings in Figure 4 were band-pass (BP) filtered in the frequency range 5 $25 \mathrm{~Hz}$. The result of doing this changed the tracings in Figure 4 only marginally and did not noticeably enhance the onset of the seizure.

Figures 7 and 8 show that the same frequency components are present in all of the depth, subdural and scalp tracings. However, the covariance between these sites, in terms of amplitude modulation, appears to be low. To examine this covariance quantitatively, correlation coefficients were calculated between the scalp recording at $\mathrm{F} 9$ and the intracranial recordings at $\mathrm{c} 4$ and $\mathrm{a} 2$ in both the pre-ictal and ictal segments. The results of these calculations are given in the Table. Given that, with $n=400$ samples, the upper $\mathrm{p}<0.01$ point for significant correlation is about 0.2 , absolute values of the numbers in the Table between 0.2 and 0.3 are interpreted as indicating weak correlation and values above 0.3 as indicating stronger correlation. Very strong correlation probably does not exist between intracranial and scalp recordings because the field of view of a scalp electrode contains many more sources than the field of view of an intracranial electrode.

An examination of the numbers in the Table indicates that, in the unfiltered recordings, there is no significant correlation between F9 on the scalp and the intracranial sites in either of the pre-ictal or the ictal segments. There is, however, strong correlation between the intracranial sites in the ictal segment. Band-pass filtering into the $5-25 \mathrm{~Hz}$ band produces no significant correlation between recordings from F9 and the intracranial sites although there is now significant correlation between the intracranial sites in the pre-ictal segment and an increase in the strength of the correlation between these sites in the ictal segment. In contrast, TP-filtering results in strong correlation between all the sites in the ictal segment. TP-8 filtering also results in significant correlation between F9 and c4 in the pre-ictal segment although the correlation between these sites in the ictal segment is strongest after TP-14 filtering. The strongest correlation in the ictal segment involving F9 occurs with a 2 after TP- 8 filtering. Also, after TP- 8 filtering, the almost complete correlation between the two intracranial sites is notable. The strength of this correlation is also evident from a visual comparison of the respective tracings in Figure 8.

A more careful examination of the numbers in the Table yields a number of interesting results related to the polarities of the correlations. Firstly, the polarities of the correlations between F9 and intracranial sites in the ictal segment are reversed after TP-8 and TP-14 filtering. Secondly, the polarity of the significant correlation between F9 and c4 seen in the pre-ictal segment is reversed in the ictal segment. Thirdly, when these filters are combined to form the TP-8-14 filter, the polarities of the correlations remain reversed but the strength of the correlations is reduced to the point where it is now not significant between F9 and $\mathrm{c} 4$.

Table: The correlation coefficients between the raw and filtered recordings from scalp electrode F9 and the subdural-electrode c4 and the depth-electrode a2 in the pre-ictal (2.4 - $4.4 \mathrm{~s})$ and ictal (6.6 - $8.6 \mathrm{~s})$ segments of the EEG.

\begin{tabular}{llll|lll}
\hline & \multicolumn{3}{c}{ Pre-ictal } & \multicolumn{3}{c}{ Ictal } \\
\cline { 2 - 7 } & F9-c4 & F9-a2 & $\mathbf{c 4 - a 2}$ & F9-c4 & F9-a2 & c4-a2 \\
Raw EEG & -0.031 & 0.152 & -0.019 & -0.074 & 0.144 & -0.577 \\
BPFilter (5-25 Hz) & 0.004 & 0.061 & -0.236 & -0.089 & 0.187 & -0.786 \\
TP-14 Filter & 0.045 & -0.041 & -0.360 & 0.424 & -0.363 & -0.790 \\
TP-8 Filter & 0.210 & 0.170 & -0.194 & -0.386 & 0.572 & -0.932 \\
TP-8-14 Filter & 0.136 & 0.096 & -0.258 & -0.175 & 0.337 & -0.899 \\
\hline
\end{tabular}

The entries in the cells illustrate the effects of the different filter characteristics. BP indicates Band Pass, TP-14 the nominally 14-15 Hz Temporal Pattern, TP-8 indicates the nominally 7-8Hz Temporal Pattern and TP-8-14 indicates the combined 7-8 and 14-15Hz Temporal Patterns. Based on 400 samples from the respective recordings for the calculations, the upper 0.01 significance point for the correlation coefficients is assumed to be about 0.2 . 


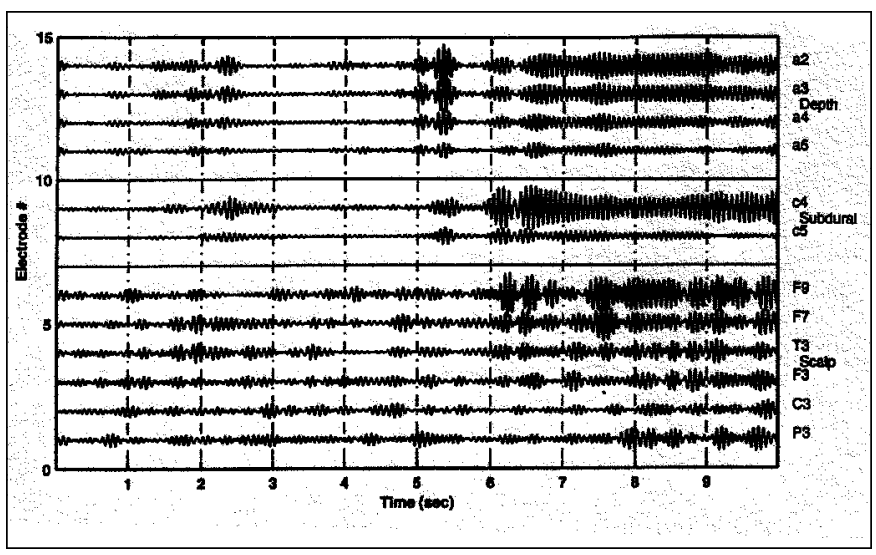

Figure 7: The raw EEG filtered with TP-14. The frequency response of this filter is shown in Figure 9A.

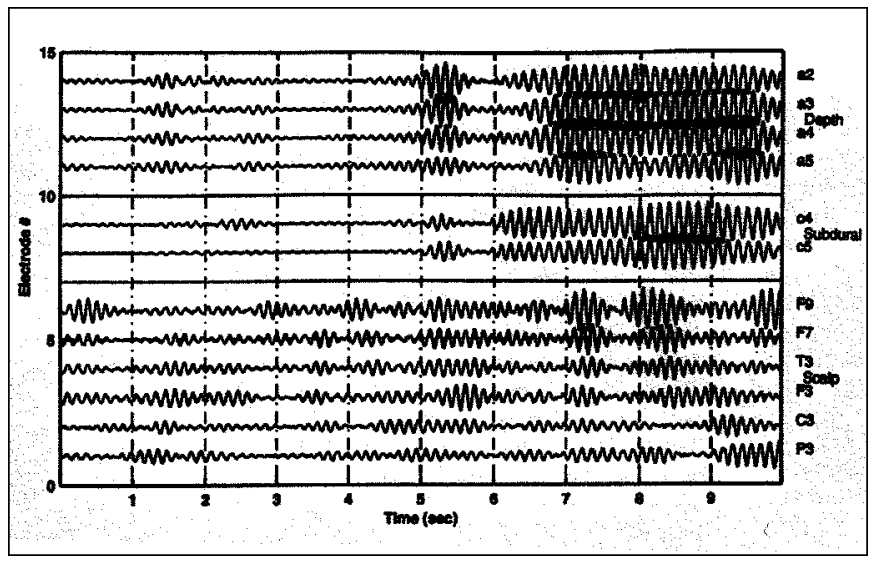

Figure 8: The raw EEG filtered with TP-8. The frequency response of this filter is shown in Figure $9 B$.

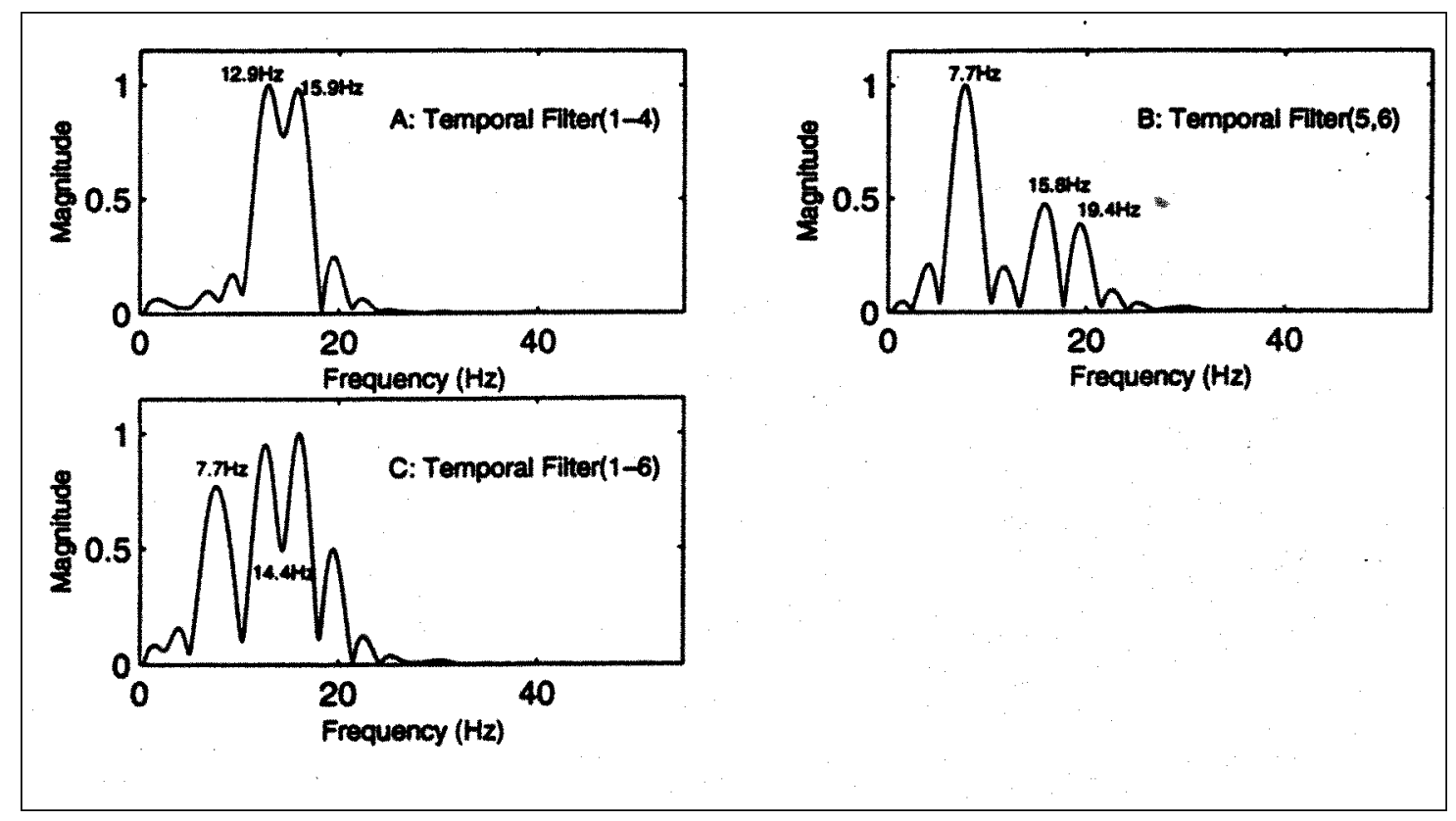

Figure 9: A. The frequency response of the filter designated as TP-14. The major peaks in this response are at 12.9 and $15.9 \mathrm{~Hz}$. $B$. The frequency response of the filter designated as TP-8. The major peaks in this response are at 7.7, 15.8 and $19.4 \mathrm{~Hz}$.

C. The frequency response of the filter designated as TP-8-14.The major peaks in this response are at 7.7, 12.9, 15.8 and 19.4 Hz.

\section{Discussion AND CONCLUSIONS}

The results of this work suggest that data-dependent TPfiltering can be a useful approach to the identification and analysis of the components of seizure onset in the EEG. The results also illustrate that TP-filtering is less effective when the pre-ictal and ictal segments contain the same frequency components. O'Neill ${ }^{21}$ has, however, shown that the detection of the seizure onset can be improved, even when this occurs, by following TP-filtering with spatial pattern (SP) filtering.

The EEG used to illustrate in detail the design and performance of the TP-filter is typical of a hippocampal seizure in which ictal activity is not obvious in the raw scalp EEG in the first phase of the seizure but is clearly manifested in depth and subdural EEGs. These results show that judiciously designed TPfilters can automatically elicit the dominant frequencies of the seizure onset in the scalp EEG. The dominant onset frequency of $7-8 \mathrm{~Hz}$ identified is consistent with the results presented by Ebersole and $\mathrm{Pacia}^{6}$ who reported the frequency range of $5-9 \mathrm{~Hz}$ for hippocampal seizures and with those of Franaszczuk et $\mathrm{al}^{26}$ who reported the frequency range of $5.3-8.4 \mathrm{~Hz}$. Other studies report dominant frequencies up to $32 \mathrm{~Hz}$ but with a mean frequency of about $16 \mathrm{~Hz} .^{2-3}$ The simplest explanation for the differences between these findings is that the 7-8 Hz component is the fundamental frequency of a hippocampal seizure recorded intracranially while the $14-15 \mathrm{~Hz}$ component arises as the first 
harmonic of the seizure waveform. The higher mean frequency of intracranial seizure reported by Javidan et $\mathrm{al}^{2-3}$ may be due to the inclusion of even more of the higher harmonics. That these higher harmonics exist in intracranial recordings is clearly evident from the spectra in Figure 6. As well, it is conceivable that in some source and/or recording configurations, one or the other of the fundamental or harmonic frequencies may not be present.

The frequency spectra shown in Figure 6 suggest that, in the ictal segment of the sample EEG, the 7-8 $\mathrm{Hz}$ and $14-15 \mathrm{~Hz}$ components in the intracranial recordings are harmonically related. Therefore, it is interesting to note that in Figures 7 and 8, in the scalp tracings, the change in the $14-15 \mathrm{~Hz}$ component is more pronounced than the $7-8 \mathrm{~Hz}$ component during the seizure onset. Due to the lack of higher frequency components in the pre-ictal EEG, the $14-15 \mathrm{~Hz}$ component appears to be a more sensitive indicator of the seizure onset than the latter. Also, a more careful examination of the independent components in Figure 5 suggests that sustained $7-8 \mathrm{~Hz}$ activity might actually begin at about $4.75 \mathrm{~s}$ in the recording and that this onset actually leads the onset of the $14-15 \mathrm{~Hz}$ activity at $6.75 \mathrm{~s}$. These observations would seem to indicate that, on the scalp, the two frequency components are, in fact, not harmonically related.

The correlation analysis between the intracranial and scalp tracings indicates that, during the ictal segment, TP-filtering results in considerably stronger correlation between the tracings than does BP filtering. Furthermore, there is stronger correlation when the two TP-filters are applied separately instead of after having been combined into one filter. Since the BPfilter is not as highly tuned to the seizure as the TP-filters, the weaker correlation is easy to explain in terms of a greater proportion of uncorrelated activity in the EEG. However, why the correlation should drop after combining two TP-filters is not so easy to explain. However, this result supports our previous speculation that the two components are, in fact, not harmonically related on the scalp. In any case, we take this finding to conclude that, in the design of TP-filters, combining independent components that do not appear to be similar should probably be avoided.

The frequency responses of the TP-filters designed in this work are such that they are optimal for simultaneously emphasizing components in the ictal segment of an EEG and deemphasizing the components in the pre-ictal segment. The critical element in the design is the selection of the segments that best characterize the seizure and background segments of the recording. The idea that the pre-ictal and ictal segments for the filter design are chosen by a human expert might be seen as a major drawback of the method. However, as reported, by Qu and Gotman, ${ }^{28}$ seizure onset patterns are highly variable between patients, the seizures may evolve from relatively subtle changes in background activity and a particular onset pattern in one patient may not lead to a seizure in another patient. It is this variability between seizures and seizure onsets that has slowed the development of a truly reliable automated seizure detector. Therefore, until the process by which seizures occur and develop is better understood, the importance of the expert epileptologist in the design of the TP-filter should not be underestimated.

To demonstrate the performance of the TP-filter in this paper, we have used only four seizures. We have, however, applied the filter to the identification of the temporal components present in the onset of many more clinical and computer-simulated seizures. This experience leads us to suggest that TP-filtering can, in fact, facilitate the identification of seizure activity in even the most problematic EEGs. Substantiation of this statement will require a quantitative analysis of these data. Nevertheless, there is little doubt that TP-filtering acts to consistently reduce recording artifact and background activity and to automatically elicit the specific temporal components present during the seizure onset. In addition, the filter design process can be extremely valuable for detecting the precise moment of seizure onset and for identifying the frequency components involved. An interesting continuation of this work will undoubtedly be a study of the utility of TP-filtering in combination with SP-filtering and source localization.

In conclusion, it would seem that TP-filtering has the potential of being a useful tool for the diagnosis of epilepsy and, more importantly, for the planning of resective surgery. The inclusion of TP-filtering in a review workstation could reduce the necessity for invasive EEG recording thereby lessening both the risk and the cost of providing this procedure to the patient. Early detection of the seizure is critical in locating the responsible source inside the brain. A developed seizure can involve large volumes of brain tissue, in effect masking the focus responsible for the onset. Since most quantitative methods of source localization assume that the sources are focal and not distributed, accurate localization of the seizure focus would almost certainly be facilitated by the early detection of the onset and the elimination of background sources not related to the seizure.

\section{APPENDIX}

The characteristics of the TP-filter, specifically the common temporal patterns, $p_{i}(m)$, and $c_{i}$, the weightings on these factors, are derived from differences in the temporal patterning of the EEG at a particular electrode site in the selected pre-ictal and ictal segments of the recording. If the sets of digital samples from these segments are depicted as $x_{a}(n)$ and $x_{b}(n)$ respectively, where $n=0$ to $T-1$, then the temporal patterning of the segments can be quantitatively characterized by their autocovariance functions. That is,

$$
R_{a}(m)=\sum_{n=0}^{T-N-1} x_{a}(n) x_{a}(n+m)
$$

and similarly for $R_{b}(m)$. The values of $R_{a}(m)$ (or $\left.R_{b}(m)\right)$ are computed for $m=0$ to $N-1$, and tend from a maximum at $m=0$, toward zero, sometimes in an oscillatory fashion, as $m$ becomes large. At $m=0, R_{a}(0)$ (or $R_{b}(0)$ ), is simply a measure of the variance of the individual samples in the segment while for other values of $m$, it is a measure of the covariance between samples separated by $m$ intervals. The TP-filter is designed from differences between the autocovariance functions of the selected pre-ictal and ictal EEG segments.

To show how this is done, let $\mathbf{R}_{\mathrm{a}}$ and $\mathbf{R}_{\mathrm{b}}$ be the temporal autocovariance matrices that correspond to the autocovariance functions of $x_{a}(n)$ and $x_{b}(n)$. Each of these matrices consists of the respective autocovariance function as its first row. Adjacent rows are formed by successively shifting the previous row circularly to the right. In this way, $N-1$ additional unique rows can be formed. The autocovariance matrices are, therefore, of 
dimension $N \times N(N \leq T)$ where $T$ is the number of digital samples in each segment.

A composite covariance matrix for the two segments is formed as:

$$
\mathbf{R}_{\mathrm{c}}=\mathbf{R}_{\mathrm{a}}+\mathbf{R}_{\mathrm{b}}
$$

From $\mathbf{B}_{\mathrm{c}}$ and $\boldsymbol{\lambda}_{\mathbf{c}}$, the $N x N$ matrices containing the eigenvectors and eigenvalues of $\mathbf{R}_{\mathrm{c}}$, a whitening transformation is constructed as:

$$
\mathbf{W}=\mathbf{B}_{\mathrm{c}} \lambda_{\mathbf{c}}^{-1 / 2}
$$

where the column dimension of $\mathbf{W}$ is the number of significant (nonzero) eigenvalues in $\lambda_{\mathbf{c}}$. This transformation is applied to $\mathbf{R}_{\mathrm{a}}$ and $\mathbf{R}_{\mathrm{b}}$ to form two whitened covariance matrices $\mathbf{S}_{\mathrm{a}}$ and $\mathbf{S}_{\mathrm{b}}$ :

$$
\mathbf{S}_{\mathrm{a}}=\mathbf{W}^{\prime} \mathbf{R}_{\mathrm{a}} \mathbf{W} \text { and } \mathbf{S}_{\mathrm{b}}=\mathbf{W}^{\prime} \mathbf{R}_{\mathrm{b}} \mathbf{W}
$$

where ' is the transpose operator. It can be shown ${ }^{28}$ that these matrices will have common eigenvectors $\mathbf{B}_{t}$ and eigenvalues $\Psi_{\text {a }}$ and $\Psi_{\mathbf{b}}$ respectively such that:

$$
\Psi_{\mathrm{a}}+\Psi_{\mathrm{b}}=\mathbf{I}
$$

where $\mathrm{I}$ is the identity matrix. Because of this constraint, those eigenvectors that account for the largest variance in $\mathbf{S}_{\mathrm{a}}$ will account for smallest variance in $\mathbf{S}_{\mathrm{b}}$ and vice versa.

From equations (A2) to (A5), it can be shown that:

$$
\begin{array}{ll} 
& \mathbf{P R}_{\mathrm{a}} \mathbf{P}^{\prime}=\Psi_{\mathrm{a}} \text { and } \mathbf{P} \mathbf{R}_{\mathrm{b}} \mathbf{P}^{\prime}=\Psi_{\mathrm{b}} \\
\text { where } & \mathbf{P}=\mathbf{B}_{\mathrm{t}}{ }^{\prime} \boldsymbol{\lambda}_{\mathrm{c}}{ }^{-1 / 2} \mathbf{B}_{\mathrm{c}}{ }^{\prime}
\end{array}
$$

is the matrix of temporal patterns common to $x_{a}(n)$ and $x_{b}(n)$. The rows of $\mathbf{P}$ that correspond to the rows in $\mathbf{B}_{\mathrm{t}}$ account for maximally different proportions of the variances in the two segments $x_{a}(n)$ and $x_{b}(n)$. Each of the rows of $\mathbf{P}$ is a temporal pattern $p_{i}(m), m=0$ to $N-1$, that simultaneously decomposes the two segments into independent components. The elements of the diagonal matrices $\Psi_{b}$ and $\Psi_{\mathbf{a}}$ are related to the variances of these components in $x_{b}(n)$ and $x_{a}(n)$ respectively. Only those rows of $\mathbf{P}$ for which $\Psi_{\mathbf{b}}>\Psi_{\mathbf{a}}$ are used for the TP-filter. The impulse response of the TP-filter is, as indicated by equation (3), the weighted sum of these patterns. In matrix terms, this sum is given by:

$$
\mathbf{h}=\left[\mathbf{C}_{q} \mathbf{P}_{q}\right]_{1}
$$

where $\mathbf{C}$ is called the matrix of temporal factors common to $x_{a}(n)$ and $x_{b}(n)$ and is given by:

$$
\mathbf{C}=\mathbf{B}_{\mathrm{c}} \boldsymbol{\lambda}_{\mathbf{c}}^{-1 / 2} \mathbf{B}_{\mathrm{t}}
$$

In equation (A8), the subscript $q$ indicates that only the $q$ rows from $\mathbf{P}$ and the corresponding $q$ columns from $\mathbf{C}$ for which $\Psi_{\mathbf{b}}>$ $\Psi_{\mathrm{a}}$ are used. The subscript 1 on the square bracket indicates that the first row of the $N x N$ matrix inside is required to form the impulse response of the filter. The other rows of this matrix are redundant since they are just circularly rotated versions of the first row.

\section{ACKNOWLEDGEMENTS}

We acknowledge the Natural Sciences and Engineering Research Council of Canada (NSERC rgpin 121524) and the Alberta Health Sciences Research Institute for their financial support and Dr. S. Spencer of the Yale School of Medicine for the data provided.

\section{REFERENCES}

1. Pacia S, Ebersole JS. Intracranial EEG substrates of scalp ictal patterns from temporal lobe foci. Epilepsia 1997; 38: 642-654.
2. Javidan M, Katz A, Tran T, et al. Frequency characteristics of neocortical and hippocampal onset seizures. Epilepsia 1992a; 33: S3-58.

3. Javidan M, Katz A, Pacia S, et al. Onset and propagation frequencies in temporal lobe seizures. Epilepsia 1992b;33:S3-59.

4. Spencer SS, Guimaraes P, Katz A, Kim J, Spencer D. Morphological patterns of seizures recorded intracranially. Epilepsia 1992; 33: 537-545.

5. Ebersole JS, Pacia SV. Temporal neocortical epilepsy syndrome: scalp EEG identification. Epilepsia 1993; 34: S7-112.

6. Ebersole JS, Pacia SV. Localization of temporal lobe foci by ictal EEG patterns. Epilepsia 1996; 37: 386-399.

7. Pacia S, Ebersole JS. The classification of temporal lobe seizures by scalp EEG. Epilepsia 1992; 33: S3-58.

8. Pacia S, Ebersole JS. Temporal neocortical epilepsy syndrome: intracranial EEG identification. Epilepsia 1993; 34: S6-26.

9. Spanedda F, Cendes F, Gotman J. Relations between EEG seizure morphology, interhemispheric spread, and mesial temporal atrophy in bitemporal epilepsy. Epilepsia 1997; 38: 1300-1314.

10. Assaf BA, Ebersole JS. Visual and quantitative ictal EEG predictors of outcome after temporal lobectomy. Epilepsia 1999; 40: 52-61.

11. Mizuno-Matsumoto Y, Okazaki K, Kato A, et al. Visualization of epileptogenic phenomena using cross-correlation analysis: localization of epileptic foci and propagation of epileptiform discharges. IEEE Trans Biomed Eng 1999; BME-46: 271-279.

12. Lopes Da Silva FH, Van Hulten K, Lommen JG, et al. Automatic detection and localization of epileptic foci. Electroencephalogr Clin Neurophysiol 1977; 43: 1-13.

13. Pfurtscheller G, Fischer G. A new approach to spike detection using a combination of inverse and matched filter techniques. Electroencephalogr Clin Neurophysiol 1978; 44: 243-247.

14. Barlow JS. EEG transient detection by matched inverse digital filtering. Electroencephalogr Clin Neurophysiol 1980;48:246-248.

15. Barlow JS. Analysis of EEG changes with carotid clamping by selective analog filtering, matched inverse digital filtering and automatic adaptive segmentation: a comparative study. Electroencephalogr Clin Neurophysiol 1984; 58: 193-204.

16. Hjorth B, Rodin E. Extraction of deep components from scalp EEG. Brain Topogr 1988a; 1: 65-69.

17. Hjorth B, Rodin E. An eigenfunction approach to the inverse problem of EEG. Brain Topogr 1988b; 1: 79-86.

18. Koles ZJ. The quantitative extraction and topographic mapping of the abnormal components in the clinical EEG. Electroencephalogr Clin Neurophysiol 1991; 79: 440-447.

19. Liu A, Hahn JS, Heldt GP, Coen RW. Detection of neonatal seizures through computerized EEG analysis. Electroencephalogr Clin Neurophysiol 1992; 82: 30-37.

20. O'Neill NS, Javidan M, Koles ZJ. Localization of seizure onset in the EEG. Proceedings of the 24th meeting of the Canadian Medical and Biological Engineering Society 1998; 14-15.

21. O'Neill NS. Temporal and spatial pattern filtering of the EEG. M.Sc Thesis, University of Alberta, Edmonton, Canada 1998.

22. Gotman J. Automatic detection of seizures and spikes. J Clin Neurophysiol 1999; 16: 130-140.

23. Osorio I, Frei MG, Wilkinson SB. Real-time automated detection and quantitative analysis of seizures and short-term prediction of clinical onset. Epilepsia 1998; 39: 615-627.

24. Mosher JC, Lewis PS, Leachy RM. Multiple dipole modelling and localization from spatio-temporal MEG data. IEEE Trans Biomed Eng 1992; BME-39: 541-557.

25. Pascual-Marqui RD, Michel CM, Lehmann D. Low resolution electromagnetic tomography: a new method for localizing electrical activity in the brain. Int J Psychophysiol 1994; 18: 49-65.

26. Franaszczuk PJ, Bergey GK, Durka PJ, Eisenberg HM. Timefrequency analysis using the matching pursuit algorithm applied to seizures originating from the mesial temporal lobe. Electroencephalogr Clin Neurophysiol 1998; 106: 513-521.

27. Qu H, Gotman J. A patient specific algorithm for the detection of seizure onset in long-term EEG monitoring: possible use as a warning device. IEEE Trans Biomed Eng 1997;BME-44:115-122.

28. Fukunaga K. Statistical pattern classification. In: Young T,. Fu KS. eds. Handbook of Pattern Recognition and Image Processing. Academic Press, 1986. 